\title{
A Novel Non-Destructive Technology for Inspecting Eggshell Cracks Using Impulsive
}

\section{Response Time}

\author{
Chung-wei Li, Jui-chun Chang, Ching-wei CHENG and Li-cheng HsieH ${ }^{*}$ \\ Department of Bio-Industrial Mechatronics Engineering, National Chung-Hsing University, 250 KuoKuang Rd., Taichung 402, Taiwan, \\ R.O.C.
}

Received February 13, 2010; Accepted September 20, 2010

An experiment system was built to measure the impulsive response signal and connected to two detectors, an accelerometer and a TCS (thickness and crack sensor). The received signals were then analyzed using the spectrum circuit, and the data were recorded. The response time of the impulse on the noncracked areas was less than $0.66 \mathrm{~ms}$, and on or near the cracked areas was above 0.82 ms. The response time recorded using accelerometer and TCS showed no significant difference, yet the TCS performed with buffer force and cheaper than accelerometer. Thus, the TCS was chosen and used in the commercial system. 400 million eggs detected for making preserved eggs, an accuracy of $97 \%$ required by the producer for this local made machine was satisfied as well. The automatic inspecting system represents the first device for both hen and duck eggs.

Keywords: eggshells cracks, eggshells thickness, acoustics, Hertz contact theory, collision theory

\section{Introduction}

Eggs form a source of nutrition in a balanced diet, alongside food such as rice and pork (Ko, 2001). In addition to their food value, eggs can be made into animal feed and fertilizers, and applied as a culture media in biology. Therefore, hen eggs and duck eggs are very important (Chang, 1994). Most eggs in Taiwan come from hens and ducks, and more than two-thirds of the total yield comes from central and southern Taiwan. The yield in Taiwan in 2008 was 6.47 billion hen eggs and 484 million duck eggs (C.O.A., 2008). The shell egg yield worldwide is more than 40 million tons, mainly from Asia. Southeastern Asia has a vast potential market for various egg products. The egg product market has a promising future worldwide.

Traditionally, egg farmers examine eggshells visually to check for surface cracks, and judge eggshell quality by lightly knocking three eggs held in two hands, and listening to the sound thus generated. Preserved-egg manufacturers also select eggs manually before making preserved-eggs. This subjective empirical egg selection process without scientific standards is prone to biased outcomes in egg selection. In ad-

*To whom correspondence should be addressed.

E-mail: lchsieh@nchu.edu.tw dition, there are three international commercial companies, MOBA in Nederland, NABEL in Japan, and DIAMOND in Canada. Their products would manage million eggs daily and are only designed for hen eggs but not for duck eggs. There is only one hen-egg processing company using MOBA's machine in Taiwan.

Many methods have been developed for measuring the quality of the fresh eggs (Ay et al., 1997; Ketelaere et al., 2000; Pai, 2000; Cho et al., 2000; Elster and Goodrum, 1991; Goodrum et al., 1992; Worley et al., 1995, Chang, 2003). Specific detecting methods are available for the eggshells. The most common method of detecting eggshell quality is the undamaged detection approach to inspect for cracks or damage. The methods used in the literatures mainly were resonant frequencies or image processing. However, the methods showed the best success rate only up to $90 \%$ to $95 \%$ which is not satisfied for the producers of preserved eggs, minimum 97\%. The inspecting speed of each method is not good enough for the producers as well.

The most common method for detecting eggshell crack in Taiwan was the undamaged detection approach. Thus, the objective of this study was to develop a detector to inspect the crack for eggs using impulsive response. It would help egg farmers to judge the quality of eggs produced by their 
poultry. The quality judgment system was based on undamaged detection and involved using the TCS (thickness and crack sensor) (Cheng, 2004) to detect cracks in the eggshells and to determine the relationship between the presence of crack and the impulsive response time.

\section{Materials and Methods}

Experimental materials Forty eggs used in this experiment were laid by a flock of commercial Single Comb White Leghorn (Hyline-36). These eggs had no visible cracks or damage to the naked eye. Therefore, the properties of eggs were considered to be physically no significant differences.

The experiment was performed with a TEKTRONIX TDS360, Tektronix, Inc., United States, double-wave digital spectrum indicator apparatus with two-wave channels, each with a capacity of 1000 samples and 8 bit vertical distinguishing rates. The largest sampling capacity was 1000 samples per sec. The analog bandwidth was up to $3.200 \mathrm{MHz}$, and the time-base was set to $2.5 \mathrm{~ns}$ per scale. The analyzing software was installed on a personal computer. The data from the spectrum apparatus indicator were recorded and analyzed, then applied in the research database.

The power supplier module for the TCS was a GW GPD3030, Good Will Instrument Co., Ltd, Taiwan. The working voltage was $2-10 \mathrm{~V}$. The accelerometer module was a KISTLER 8704B100M1, Kistler Instrument AG, Switzerland. Voltage sensitivity: $49.36 \mathrm{mV} / \mathrm{g}$, Frequency range: 20-10000 $\mathrm{Hz}$ ) which is a pressing-electricity accelerometer to detect the impulse response time. Figure 1 illustrates the accelerometer and the signal amplifier, module DYTRAN 4105C, Dytran Instruments Inc., United States. If the voltage signal was weak, then the signal amplifier magnified it in order to observe the signals received.

TCS was Taiwan device patent designed for inspection of vegetables and undamaged eggs, which is a detector for maturity, moisture, elasticity and the internal quality of vegetables, and detect eggshell cracks or damage, respectively.

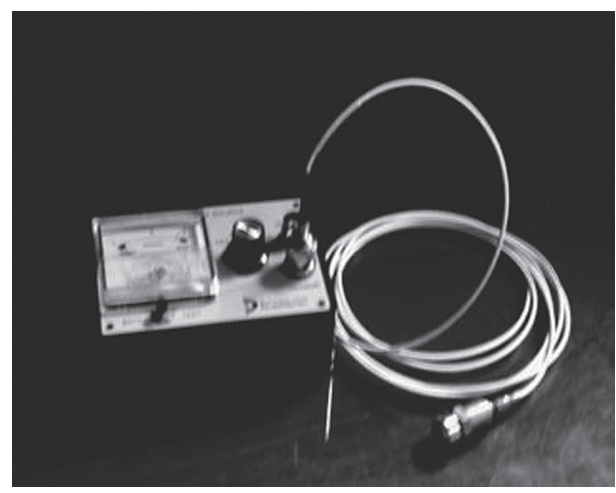

Fig. 1. Accelerometer and the signal amplifier.
The sensor works according to acoustics theory. A sound is produced after the hermetically sealed sensor head knocks the egg shell. The wave is then sent to the tiny microphone by the aluminum sensor head. The microphone module was a Votronic SH0526-315, Votronic CES Technology GmbH, Germany. Because the sealed cap is fixed onto the tube, a buffer to the counter reaction is generated in order to counter the homo-polar ring-shaped magnet on the sealed cap and the movable components. Consequently, buffering the eggshells from the counter force avoids damage, as shown in Fig. 2.

Since the eggshell has a thin shell structure, the contact deformation is caused not only by elastic deformation of eggshell material, but also by deflection of eggshell like thin plate deflection. In addition, the eggshell has been shown as homogeneous and elastic continuum material (Lin et al.,

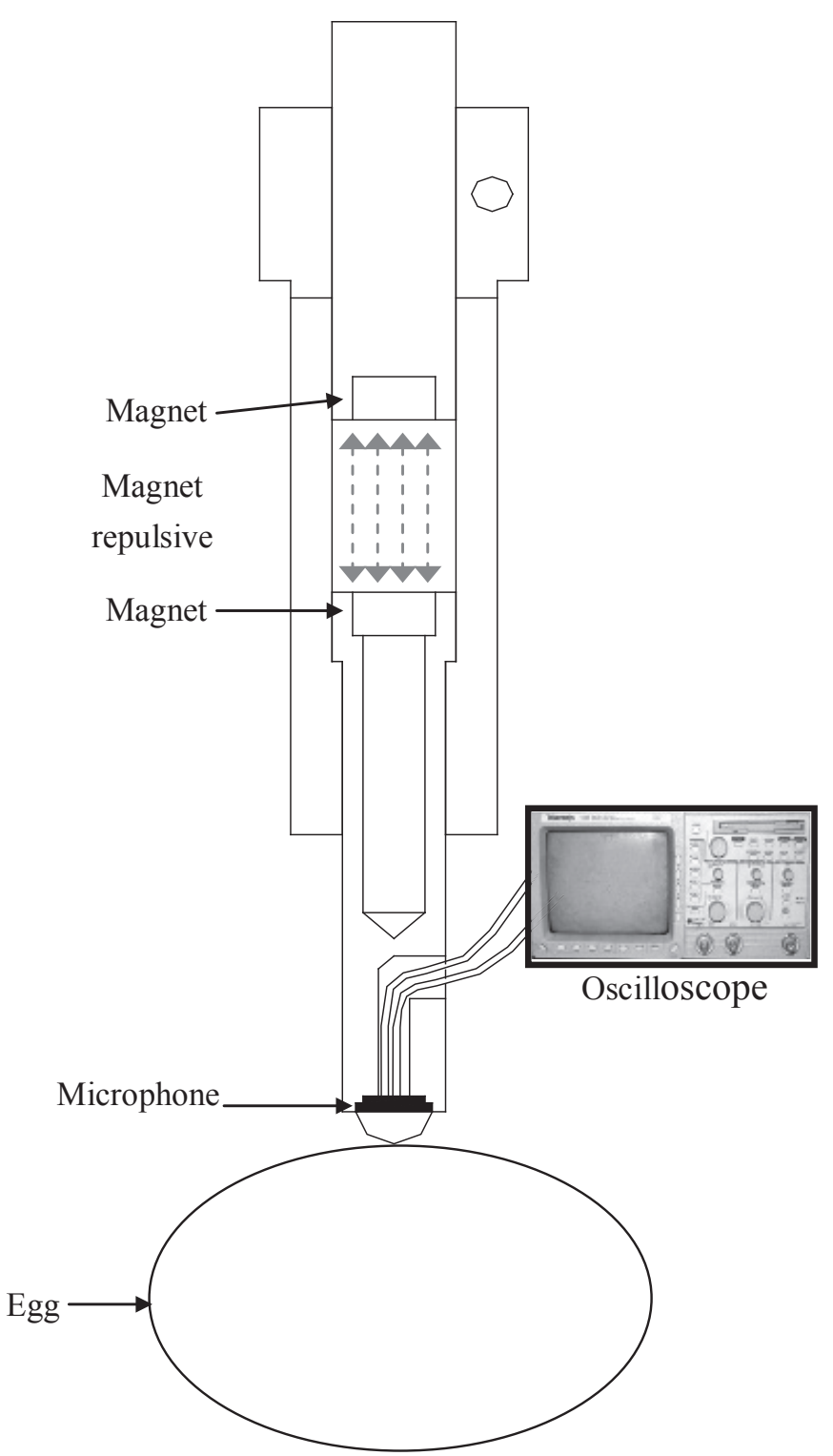

Fig. 2. Illustration of TCS, using magnet as a damper to knock an egg, and collecting signal by microphone and oscilloscope. 


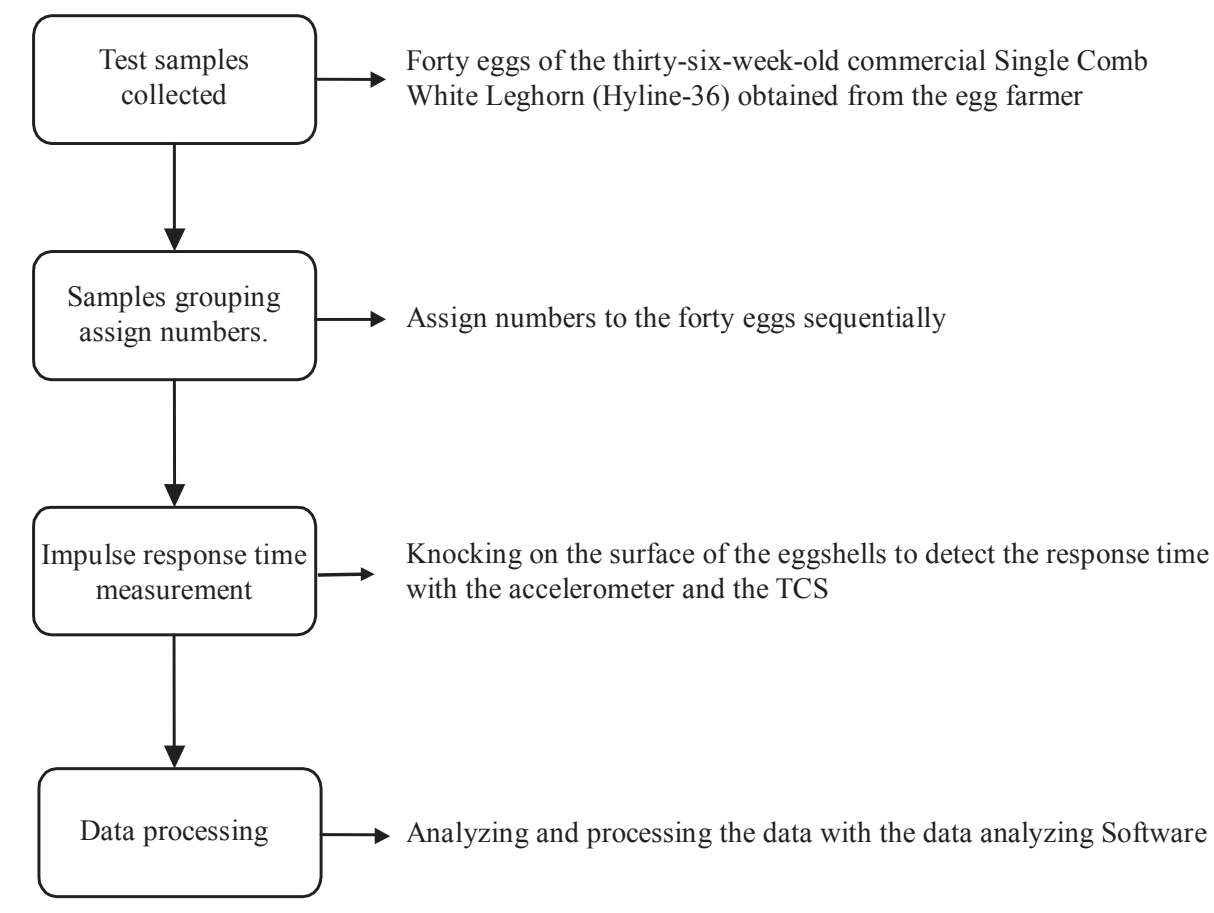

Fig. 3. Experimental flow chart.

1995; Lin et al., 1996; Kitching, 1997; Coucke et al., 1998). In this research, eggshell deformation is treated as an elastic category in Hertz contact theory.

Experimental method According to Chang (2003), the impulse time and the thickness of the eggshell had a strong negative relationship $\left(R^{2}=0.86, p<0.05\right)$. Using accelerometer and TCS, to inspect the eggshell thickness and determine the crack associated with impulsive response time is possible. The method or procedure used in this study was followed as collecting sample eggs, numbering the samples, inspecting, and analyzing the results. A flow chart is shown in Fig. 3.

For inspection, test points should be defined or determined to secure the results. First of all, since the shape of the egg is elliptical similarly, each test point picked at the same curvature would have a consistent eggshell thickness. The test points were set so that they were comprehensive and with no omission of crack points. Accordingly, the equator was initially taken as the cross axle; four longitude lines were drawn $90^{\circ}$ apart so that the longitudinal lines crossed the axle at four points. Each egg thus had 6 test points in total, including the two end points of the longitudinal lines, as shown in Fig. 4, (a). The preliminary experimental result shows that there was no distinctive difference among the four test points scattered on the equators for shell thickness determination. The experiment has a confidence level under $95 \%(p>0.05)$. The response time of the impact impulse of the four points remained consistent. Thus, the assumption of

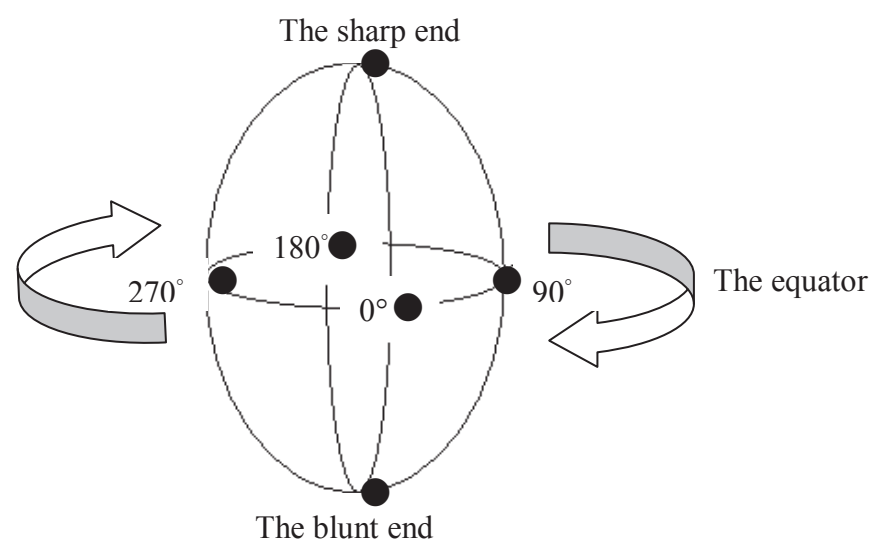

(a)

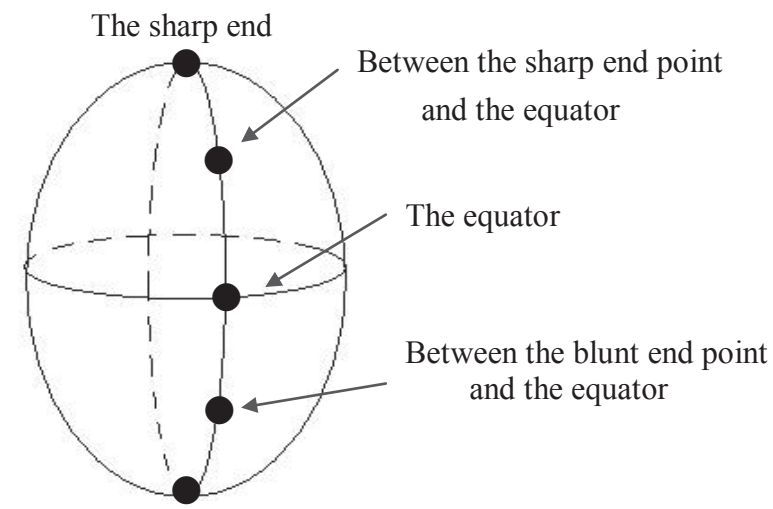

The blunt end

(b)

Fig. 4. Test point schematic diagrams. (a) Original test points, (b) Modified test points. (' $'$ ' represents the test points.) 
the consistent eggshell thickness in one curvature was confirmed. The test points were then modified, taking either longitudinal line crossed with the equator circumference as the equator point. The pointed end of the egg was taken as the second designated point. The third re-designated point was exactly midway between the sharp end point and the equator. The blunt end of the egg was taken as the fourth designated point. The last point was re-designated as the point between the blunt end and the equator point. Finally, five points were designated in total for this study (Fig. 4, (b)).

Once the test points were determined, the knocking method using either accelerometer or TCS would be applied on them. Since the TCS was a new device, accelerometer was a popular measuring device and was adequate to confirm the accuracy of TCS. To measure correctly the response time of the impulse, the eggs were placed onto the egg racks to prevent errors from hard impact resulting from movement. The accelerometer and the TCS were linked to the spectrum indicator. The TCS has two magnets. Since using magnet repulsive, the sensor's head speed doesn't need to be kept constant for every knocking of egg. The eggs were then held and knocked directly onto the five test designated points, and the impact response time was recorded (Fig. 5, (a)). During the experiment process, it was a must to control the output voltage to prevent errors resulting from different impact strengths. According to signals that were too weak for observation and analysis were magnified using the signal amplifier. Finally, the signals were obtained and were computed by the spectrum indicator circuit. The data were then saved and recorded in the database for further analysis.

\section{Results}

This experiment employed both the TCS and accelerometer methods to pose impacts on the eggs in order to measure the impact response signals. Experimental results (Table 1)
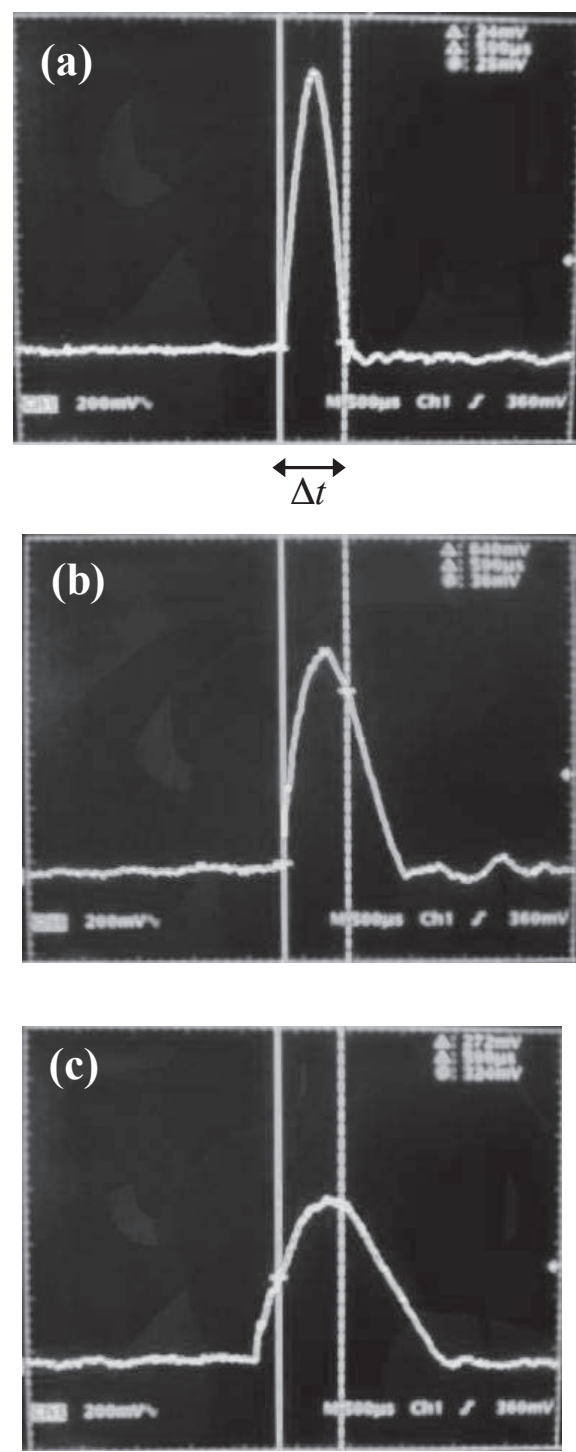

Fig. 5. Impulse impact response time. (a) illustration for signal of impulsive response and for non-crack eggshell in the width between two lines $(\Delta t)$, (b) signal of impulsive response for near the crack to go beyond the scope $(\Delta t)$, (c) signal of impulsive response on the crack to go beyond the scope $(\Delta t)$.

Table 1. The analyzed impulse impact response time using different detectors at various test points.

\begin{tabular}{|c|c|c|c|c|c|c|}
\hline Items & $\begin{array}{l}\text { The sharp end } \\
\text { (ms) }\end{array}$ & $\begin{array}{l}\text { Between } \\
\text { the sharp end point } \\
\text { and the equator } \\
(\mathrm{ms})\end{array}$ & $\begin{array}{l}\text { The equator } \\
\text { (ms) }\end{array}$ & $\begin{array}{l}\text { Between } \\
\text { the blunt end point } \\
\text { and the equator } \\
(\mathrm{ms})\end{array}$ & $\begin{array}{l}\text { The blunt end } \\
\text { (ms) }\end{array}$ & $\begin{array}{l}\text { Average } \\
\quad(\mathrm{ms})\end{array}$ \\
\hline $\begin{array}{l}\text { non-crack eggs } \\
\text { using accelerometer }\end{array}$ & $0.47 \pm 0.05$ & $0.56 \pm 0.04$ & $0.61 \pm 0.04$ & $0.56 \pm 0.04$ & $0.55 \pm 0.04$ & $0.55 \pm 0.04$ \\
\hline $\begin{array}{c}\text { non-crack eggs } \\
\text { using TCS }\end{array}$ & $0.49 \pm 0.05$ & $0.54 \pm 0.04$ & $0.61 \pm 0.05$ & $0.57 \pm 0.04$ & $0.56 \pm 0.04$ & $0.55 \pm 0.03$ \\
\hline $\begin{array}{l}\text { crack eggs } \\
\text { using TCS }\end{array}$ & $0.88 \pm 0.05$ & $1.01 \pm 0.03$ & $1.07 \pm 0.07$ & $1.04 \pm 0.04$ & $0.98 \pm 0.04$ & $0.99 \pm 0.02$ \\
\hline
\end{tabular}

\footnotetext{
${ }^{*}$ Values are means \pm SD $(n=40)$.
} 
indicate that the pointed end of the eggs had the shortest response time of impact, followed by the blunt end, while the longest response time of impact occurred at the equator circumference. With the coefficient of variation at a confidence level of $95 \%$, the impulse response time associated with two pairs of locations shows that the sharp end to the blunt end and the sharp end to the equator point exhibited a clear difference for both location combinations.

Figure 6 shows the correlation of two sensors, accelerometer and TCS, when collected the impulsive response time with non-crack eggshells at various test points. The correlation coefficients are $0.903,0.918,0.842$ and 0.939 for the sharp end, the equator, the blunt end, and the average for entire eggshell, respectively. Additionally, the results analyzed with coefficient variation at $95 \%$ confidence level indicate that the impulse impact response time accumulated by the two sensors from the eggshells were not significantly different $(p>0.05)$. Apparently, the new detector, TCS, performed as well as accelerometer. Furthermore, the signal of the two sensors to detect the eggshell knocking process was stable.

To differentiate whether the eggshell was damaged, a 6-10 mm crack on each of 10 perfect eggshells was knocked randomly at 5 different locations. Lin et al. (2001) found that the effective range was about 20 to $30 \mathrm{~mm}$. Therefore, the conservative estimate was made to choose $20 \mathrm{~mm}$ as an effective measuring range. The results of detecting the response time with TCS for damaged eggs near the crack 15 $\mathrm{mm}$ were compared to that of non-cracked areas (Table 1). The response time of the impulse on the non-cracked areas and on or near the cracked areas were found to be less than $0.66 \mathrm{~ms}$ and above $0.82 \mathrm{~ms}$, respectively.

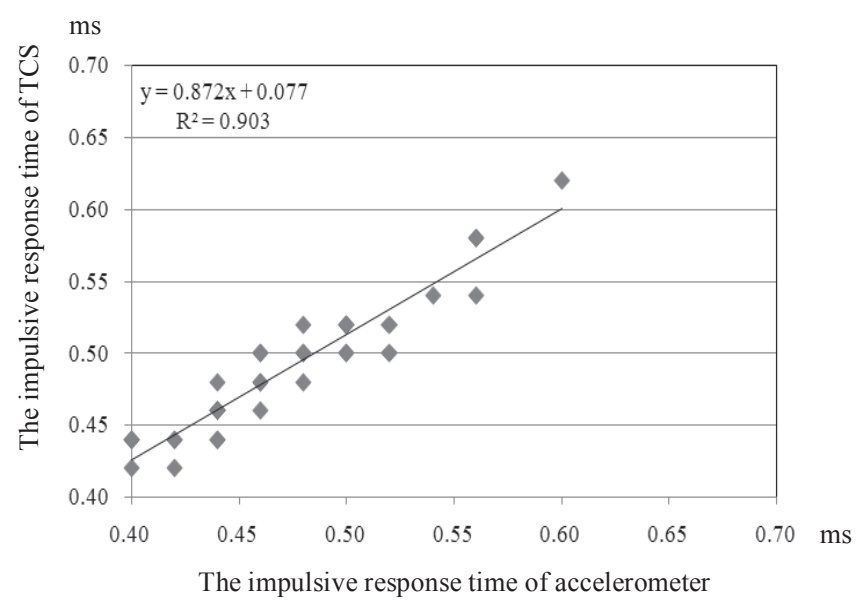

(a)

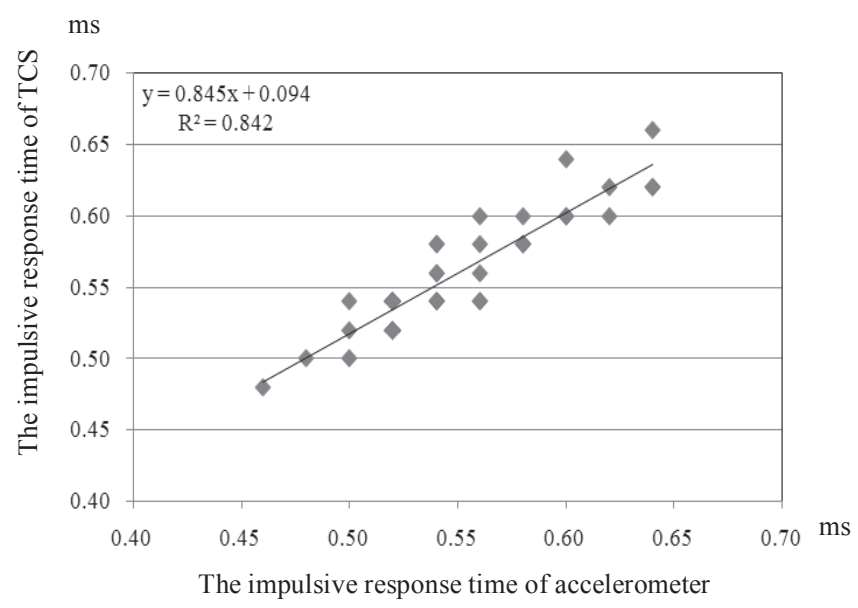

(c)

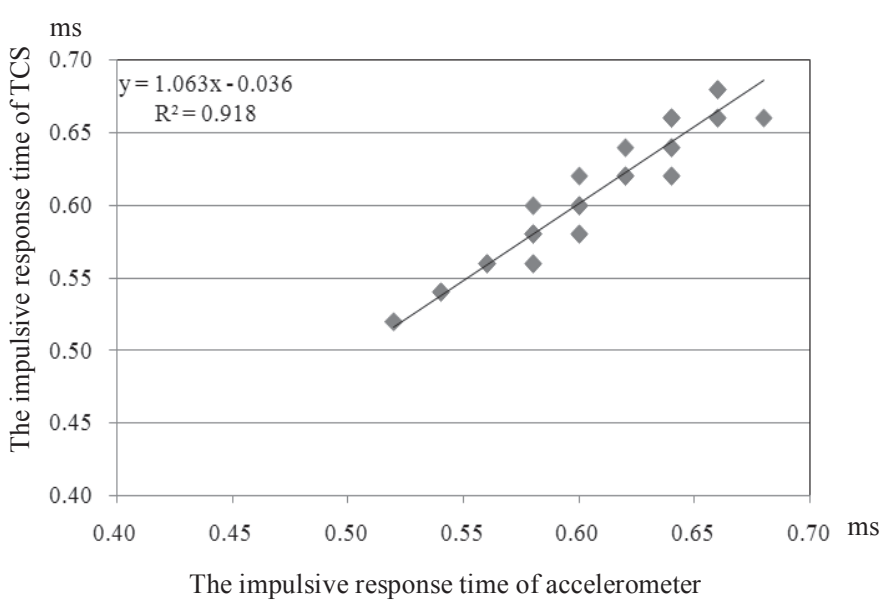

(b)

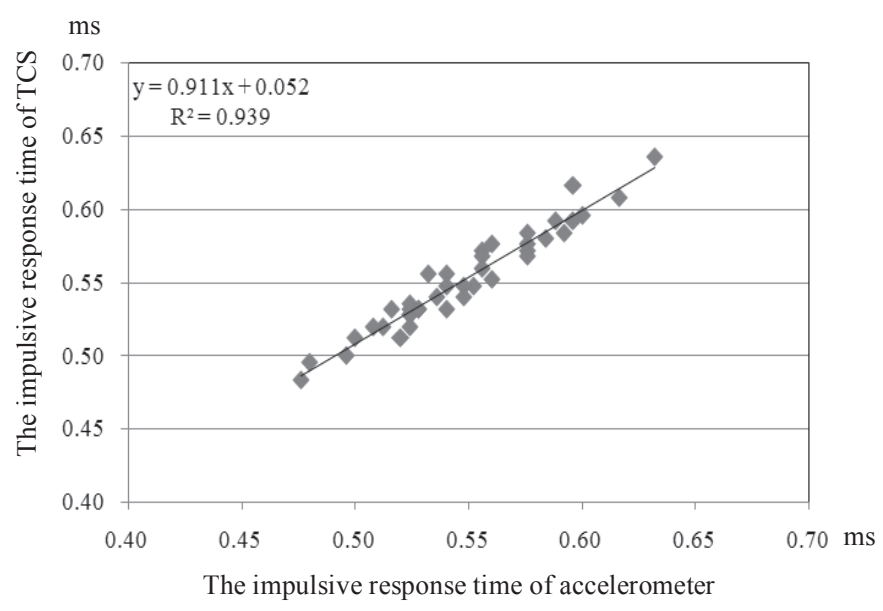

(d)

Fig. 6. Correlation of accelerometer and TCS with the impulsive response time recorded at various test points. (a) the sharp end, (b) the equator, (c) the blunt end, (d) the average of the test points. 


\section{Discussion}

Narushin (1997) found that the thickness of an eggshell can be measured from the weight of the egg or nondestructive compression deformation methods, implying that thickness of an eggshell can also be predicted indirectly from these variables. Voisey et al. (1979) concluded that the compression deformity is negatively linked to the compression damage; i.e. higher compression damage is correlated with lower compression deformity. Thus, the strain $(\varepsilon)$ decreases accordingly. From Hook's Law, Young's Modulus $(E)$ is given by the stress divided by strain $(E=\sigma / \varepsilon)$; therefore, a thicker eggshell leads to a larger value for Young's Modulus.

The Hertz contact theory (Timoshenko et al., 1975) implies that a higher $E$ value in Young's Modulus leads to a smaller constant $k$. In addition, from collision theory and Newton's law, it can be predicted that a thicker eggshell leads to less deformity and a short contact duration during collision when the sensors constantly knock on the testing points of the eggshell. Conversely, if the eggshell is thin, then collision causes high deformity, and contact duration during collision is long. Thus, the thickness of the eggshell is negatively linked to the impulse response time. If the sensor knocks on a crack in the eggshell, then the impulse response time obviously lengthens, since the crack significantly lowers the overall Young's Modulus of the egg, varying the hardness of the egg structure. The elastic collision of spheres in the Hertz contact theory states that a lower value of Young's Modulus raises the constant $k$ value. A larger $k$ raises the compress distance when spheres collide, thus lengthening the contact time. Sensor knocking on an eggshell crack produces similar results to a thinning eggshell, with a much longer impulse response time than that of an intact area (Fig. 5).

The impulse response time of the different test points was quite variable. The sharp ends of the Single Comb White Leghorn eggs had an average thickness of $0.358 \mathrm{~mm}$. The average thickness was $0.347 \mathrm{~mm}$ at the equator circumference, and $0.338 \mathrm{~mm}$ at the blunt end. The difference in average eggshell thickness among the three test points was small (about $0.02 \mathrm{~mm}$ ). The impulse response time detected by TCS was $0.49 \mathrm{~ms}$ at the sharp end, $0.61 \mathrm{~ms}$ at the equator circumference, and $0.56 \mathrm{~ms}$ at the blunt end, revealing a clear difference among the three regions. This data analysis indicates that the sharp end of an egg has the thickest shell but the shortest impulse response time. The equator circumference of an egg has a thickness between the sharp and blunt ends, but has the longest impulse response time. The blunt end of an egg has the thinnest shell, and its impulse response time is between those of the sharp end and the circumference area.

The elastic collision of spheres explains that, if the col- lision of the sensor with the egg surface is taken as the collision of two spheres, then contact pressure develops at the contact point when the head of the sensor touches the egg surface. However, the relative velocity when the sensor head first touches the egg is assumed to be equivalent, and the eggshell substance is identical, then the variation in the sensor head with Young's Modulus and Poisson ratio is not significant. The only consideration in this case is the difference in curvature radius of the sharp end, equator circumference and the blunt end of an egg, causing the collision time to vary.

The compression distance $(\alpha)$ is positively related to collision time. Additionally, the product of constants $n$ and $n_{1}$ $(\sqrt{\mathrm{cm}} / \mathrm{kg})$ is negatively linked to the compression distance during collision. Assuming the radii of the sensor head and the egg are $R_{1}$ and $R_{2}$, respectively, then

$$
n \times n_{1}=\sqrt{\frac{16}{9 \pi^{2}} \frac{R_{1} R_{2}}{\left(R_{1}+R_{2}\right)}} \times \frac{3}{4 \pi} \frac{R_{1}^{3} \rho_{1}+R_{2}^{3} \rho_{2}}{R_{1}^{3} \rho_{1} R_{2}^{3} \rho_{2}}
$$

Equation 1 is referred to Eq. A12. The density of the sensor is given as $\rho_{1}$. The mass exerted on the egg surface, when the sensor head touches the egg, results from both the impact of the sensor head and the moving parts of the sensor head. Therefore, the mass of the moving parts of the sensor head needs to be addressed. The total density of the sensor head is then determined from the mass and volume. Calculation results show that the total density of the sensor head is approximately $4.79 \times 10^{4} \mathrm{~kg} / \mathrm{m}^{3}$, and that the density of the eggshell is given as $\rho_{2}$. Experiments on egg products indicate that the density of eggshells is approximately $1.18 \times 10^{3} \mathrm{~kg} /$ $\mathrm{m}^{3}$; when the density of the two is entered into Equation 1, and the test radius on the egg $\left(R_{2}\right)$ increased, then the product of $n$ and $n_{1}$ decreases relatively (Fig. 7, (a)), creating a negative relationship. The product variants of $n$ and $n_{1}$ decrease as $R_{2}$ increases. Analyzed results show that the product of $n$ and $n_{1}$ is inversely proportional to the highest compress distance during collision $\left(\alpha_{1}, \mathrm{~mm}\right)$. These findings indicate that increasing the test radius $\left(R_{2}\right)$ of the egg also increases the highest compression distance during collision, because of the relative rise in contact time (Fig. $7,(\mathrm{~b})$ ).

Figure 8 shows a sketch of the shape of an egg. The sharp end has the smallest radius, followed by the blunt end, and the equator circumference is the largest; i.e. $r_{3}>r_{2}>r_{1}$. These findings imply that the contact time is the longest at the equator circumference, followed by the blunt end, and the sharp end has the shortest contact time.

The overall results of this experiment reveal that changes in the shape of the egg between the test points may cause changes in the impulse response time. A point with a smaller egg radius produces a larger variation in the compressed 


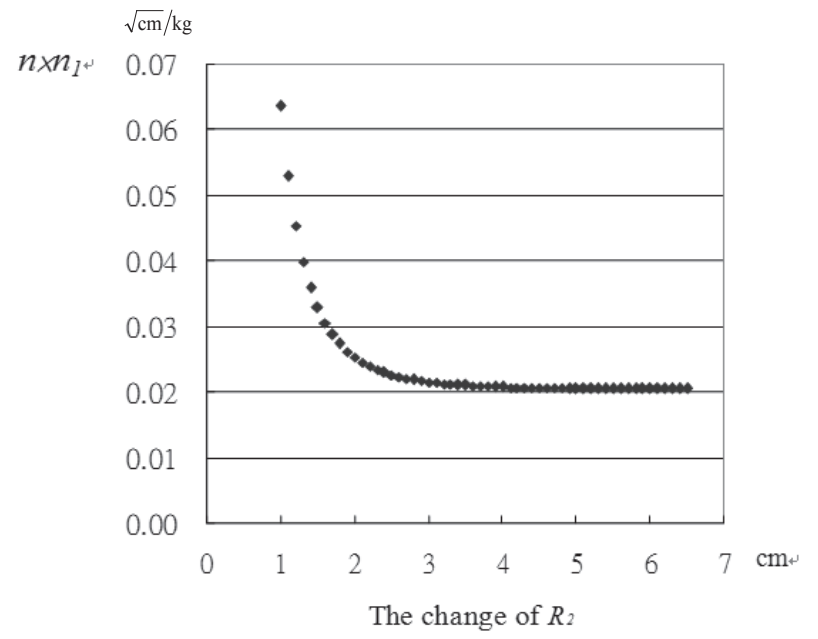

(a)

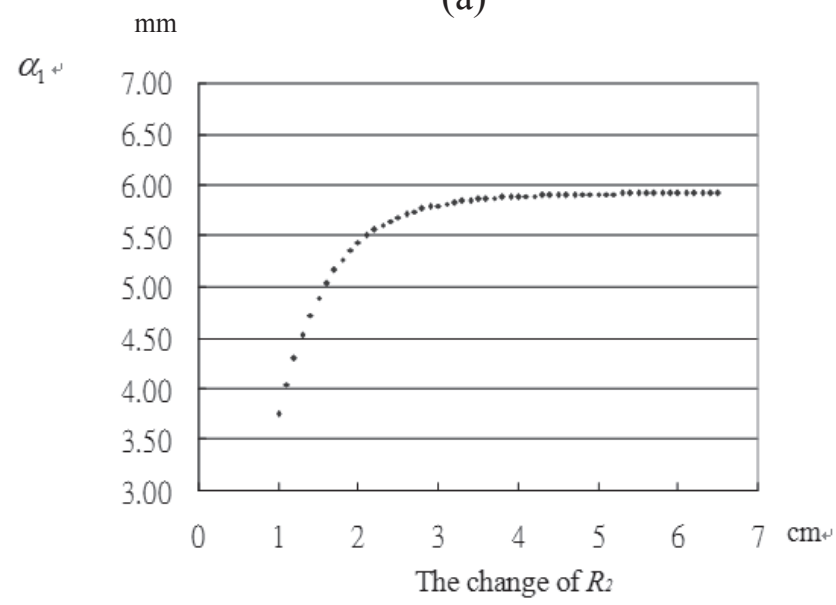

(b)

Fig. 7. (a) The relationship of change of $R_{2}$ and $n \times n_{1}$, (b) The relationship of change of $R_{2}$ and $\alpha_{1}$.

distance, as shown in Fig. 7b. The variation decreases as the radius rises. If the relationship between the thickness of the eggshells and the changes in the shape end of the egg is considered when measuring the impulse response time, then the modulus $\left(R_{2}\right)$ of the sharp-end thickness of the eggshell is lowered, because of the smaller radius of the sharp end $\left(R_{2}\right)$. A small change to the radius produces a big change in the compression distance $\left(\alpha_{1}\right)$ during contact, affecting the contact time. The radius of the sharp end of an egg has a greater influence than the thickness of the eggshell on the impulse response time.

The impulse time varies obviously with spot of knocking when the eggshell has uniform thickness. This is explained by the elastic collision of spheres in the Hertz contact theory. The curvature radius of the three test spot of the eggs, i.e. the sharp end, equator circumference and the blunt end, were different. The contact time rises relatively to the increase in radius of the test spot on the egg when the sensor knocks the

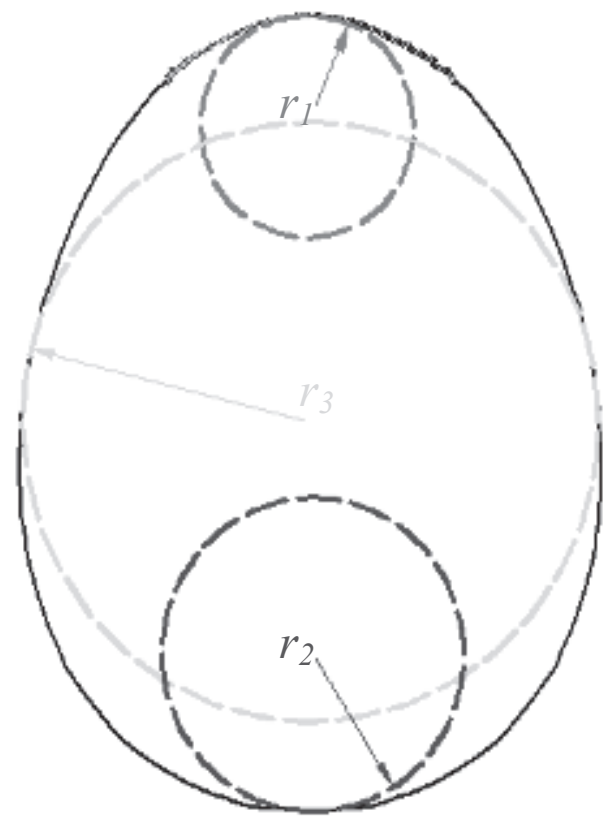

Fig. 8. The geometry of an egg for the sharp end $\left(r_{1}\right)$, the blunt end $\left(r_{2}\right)$, and the equator $\left(r_{3}\right)$.

egg. With that, the impulse response time data indicate that the response time is the longest at the equator, followed by the blunt end the second, and the sharp end has the shortest response time.

The accelerometer acted as a rigid body without buffer force. If the knocking force was not accurately controlled, it would create even more cracks. On the contrary, the TCS solved this problem by using the magnetic repulsion and lessoned the knocking force. In addition, the cost of the TCS was about $90 \%$ lower than which of the accelerometer. According to the research, the signal of eggshell knocking was the best if within the radius of $20 \mathrm{~mm}$. As a result, in planning the automatic inspection system, each machine should be assembled with 56 knocking sensors, which would increase manufacturing cost tremendously. Thus, the TCS was chosen and adopted for the tests. The device for detecting the egg crack is mainly developed for the egg farmer producing 50000 eggs daily for factories in Taiwan. The TCS has been installed in an automatic inspecting machine and over 20 commercial machines were sold in Taiwan. The highest frequencies of these market machines have been applied over 50 million times. In other words, each machine has been applied for 20 million times. Furthermore, over 400 million eggs have been applied using this device, not only the hen eggs but also duck eggs, which is the only machine implemented for both products in the world. An accuracy of $97 \%$ required by the producer for this local made machine was satisfied as well. 


\section{Conclusion}

This study adopted two different sensors, namely the TCS and the accelerometer, using Hertz contact theory to knock the egg surface in order to measure the impulse response time which was then analyzed and compared with the thickness of the eggshell. Analysis results also indicate that the response time at the intact surface was less than $0.66 \mathrm{~ms}$ and a response time exceeding $0.82 \mathrm{~ms}$ as knocking around cracks or on the cracks gave. This can be used as an easy identification of a crack in the both hen and duck eggs. TCS can thus be applied to develop an automatic inspecting system for eggshell cracks especially for duck eggs.

Acknowledgements This work was supported by Council of Agriculture, Executive Yuan, Taiwan R.O.C., and the authors gratefully thank to supporter. This invention attained The 2007 Award for Outstanding Contributions in Science and Technology by National Science Council, Taiwan R.O.C.

\section{Appendix}

Voisey et al. (1979) found that the thickness of an eggshell can be measured from the weight of the egg or undamaged compression deformation methods, implying that thickness of an eggshell can also be predicted indirectly from these variables. According to Voisey et al. (1979), the thickness of an eggshell $(T)$, the compression deformity (Def) and the estimated compression damage are related by the following equation:

$$
\begin{aligned}
& F=-1022+137 T \\
& F=5794-3.3 D e f
\end{aligned}
$$

In these equations, $F$ represents the compression damage (g); T represents the thickness of the eggshell $(\mu \mathrm{m})$, and Def represents the compression deformity $(\mu \mathrm{m})$. Equation A1 indicates that the thickness of the eggshell is directly linked to the compression damage, i.e. a thicker eggshell leads to greater compression damage. Extracting the results of Eq. A1 into Eq. A2 shows that compression deformity falls with increasing compression damage. If the surface area $(A)$ and the longitude $(L)$ of the compression are fixed, then the pressure in the affected surface area (i.e. stress, $\sigma)$ rises $(\sigma=F / A)$ when the eggshell is exposed to greater compression damage. Accordingly, the compression deformity is negatively linked to the compression damage; i.e. higher compression damage leads to lower compression deformity. Thus, the strain $(\varepsilon)$ decreases accordingly $(\varepsilon=\delta / L)$. From Hook's Law, Young's Modulus $(E)$ is given by the stress divided by strain $(E=\sigma / \varepsilon)$; therefore, a thicker eggshell leads to a larger value for Young's Modulus.

The Hertz contact theory (Timoshenko et al., 1975) im- plies that a higher $E$ value in Young's Modulus leads to a smaller constant $k$ (Eq. A3). According to collision theory, two colliding spheres would come into contact at a point. Given that the mass of each sphere is $m_{1}$ and $m_{2}$, respectively, the relative velocity of the spheres when the spheres start to collide is $V$, and the distance between the centers of the two spheres gradually declines after the collision. Additionally, the motion velocity gradually declines until the sphere comes to a complete stop. This is the condition in which the spheres are most compressed after collision. The spheres then gradually resume their original shapes, and detach.

$$
k_{1}=\frac{1-v_{1}^{2}}{\pi E_{1}} \quad k_{2}=\frac{1-v_{2}^{2}}{\pi E_{2}}
$$

Contact pressure $P$ is generated when the spheres collide, reducing the contact motion velocity. At this point, the velocity of each sphere is $V_{1}$ and $V_{2}$, respectively, as indicated by Newton's law

$$
m_{1}=\frac{d V_{1}}{d t}-P \quad m_{2}=\frac{d V_{2}}{d t}-P
$$

can get

$$
n=\sqrt{\frac{16}{9 \pi^{2}} \frac{R_{1} R_{2}}{\left(k_{1}+k_{2}\right)^{2}\left(R_{1}+R_{2}\right)}}, \quad n_{1}=\frac{m_{1}+m_{2}}{m_{1} m_{2}}
$$

thus

$$
P=n a^{3 / 2}
$$

$$
\ddot{\alpha}=-n n_{1} \alpha^{3 / 2}
$$

$1 / 2 d(\dot{\alpha})^{2}=-n n_{1} \alpha^{3 / 2} d \alpha$

$$
1 / 2\left(\dot{\alpha}^{2}-V^{2}\right)=-2 / 5 n n_{1} \alpha^{5 / 2}
$$

In the above equation, $V$ represents the relative velocity when the spheres first come into contact with each other. After collision, when the spheres are most compressed, the relative velocity is zero, which also means that $\dot{\alpha}=0$. The equations above can be utilized to determine the distance between the spheres when they are most compressed $\left(\alpha_{1}, \mathrm{~mm}\right)$, as in Eq. A9. The time required for the two spheres to collide can be derived from Eq. 8, as shown in Eq. A10.

$$
\begin{aligned}
& \alpha_{1}=\left(\frac{5}{4} \frac{V^{2}}{n n_{1}}\right)^{2 / 5} \\
& d t=\frac{d \alpha}{\sqrt{V^{2}-4 / 5 n n_{1} \alpha^{5 / 2}}}
\end{aligned}
$$

$\alpha / \alpha_{1}=x$ ' by employing Eq. A9 the above can be simplified to 


$$
d t=\frac{\alpha_{1}}{V} \frac{d x}{\sqrt{1-(x)^{5 / 2}}}
$$

Thus, the collision time of the spheres is

$$
t=\frac{2 \alpha_{1}}{V} \int_{0}^{1} \frac{d x}{1-(x)^{5 / 2}}=2.94 \frac{\alpha_{1}}{V}
$$

Equation A5 - A9 indicate that $k$ affects the distance of the spheres when they are compressed. A smaller $k$ means a larger Young's Modulus, implying that the spheres deform least when they collide, which is when the distance between them when they compress is the smallest, and the contact time during collision is also the shortest. Conversely, given a large $k$, the spheres are easily deformed when they collide, leading to a long contact time during collision.

In conclusion, a thicker eggshell leads to less deformity and a short contact duration during collision when the sensors constantly knock on the testing points of the eggshell. Conversely, if the eggshell is thin, then collision causes high deformity, and contact duration during collision is long. Thus, the thickness of the eggshell is negatively linked to the impulse response time. If the sensor knocks on a crack in the eggshell, then the impulse response time obviously lengthens, since the crack significantly lowers the overall Young's Modulus of the egg, varying the hardness of the egg structure. The elastic collision of spheres in the Hertz contact theory states that a lower value of Young's Modulus raises the constant $k$ value in Eq. A6b. According to Eq. A5 - Eq. A9, a larger $k$ raises the compress distance when spheres collide, thus lengthening the contact time. Sensor knocking on an eggshell crack produces similar results to a thinning eggshell, with a much longer impulse response time than that of an intact area.

The impulse response times of the different test points were quite varied. The sharp ends of the White Leghorn chicken eggs had an average thickness of $0.358 \mathrm{~mm}$. The average thickness was $0.347 \mathrm{~mm}$ at the equator circumference, and $0.338 \mathrm{~mm}$ at the blunt end. The difference in average eggshell thickness among the three test points was thus very small, at about $0.02 \mathrm{~mm}$. The egg quality sensor measured the impulse response time as $0.49 \mathrm{~ms}$ at the sharp end, 0.61 $\mathrm{ms}$ at the equator circumference, and $0.56 \mathrm{~ms}$ at the blunt end, revealing a clear difference among the three regions. This data analysis indicates that the sharp end of an egg has the thickest shell but the shortest impulse response time. The equator circumference of an egg has a thickness between the sharp and blunt ends, but has the longest impulse response time. The blunt end of an egg has the thinnest shell, and its impulse response time is between those of the sharp end and the circumference area.
The elastic collision of spheres explains that if the collision of the sensor with the egg surface is taken as the collision of two spheres, then contact pressure develops at the contact point when the head of the sensor touches the egg surface. Equation A9 indicates that the variables that affect the compress distance when two objects touch are the relative velocity at contact $(V)$, the physical characteristics of the sphere built (the Young's Modulus, the Poisson's ratio and the radius of the spheres) and the mass of the spheres, shown as $n$ and $n_{1}$ in Eq. A9. However, the relative velocity when the sensor head first touches the egg is assumed to be equivalent, and the eggshell substance is identical, then the variation in the sensor head with Young's Modulus and Poisson's ration is not important for calculating constant $n$. The only consideration in this case is the difference in curvature radius of the sharp end, equator circumference and the blunt end of an egg, causing the collision time to vary.

The compression distance is positively related to collision time, according to Eq. A11. Additionally, the product of constants $n$ and $n_{1}(\sqrt{\mathrm{cm}} / \mathrm{kg})$ is negatively linked to the compression distance during collision, according to Eq. A9. Constant $n_{1}$ represents the reciprocal sum of the sphere mass as in Eq. A10, where $m_{1}$ and $m_{2}$ denote the mass of the sensor head and the egg, respectively. Assuming the volume of the sensor head and the egg are $R_{1}$ and $R_{2}$ respectively as the radius of the spheres, then

$$
n \times n_{1}=\sqrt{\frac{16}{9 \pi^{2}} \frac{R_{1} R_{2}}{\left(R_{1}+R_{2}\right)}} \times \frac{3}{4 \pi} \frac{R_{1}^{3} \rho_{1}+R_{2}^{3} \rho_{2}}{R_{1}^{3} \rho_{1} R_{2}{ }^{3} \rho_{2}}
$$

The density of the sensor is given as $\rho_{1}$. The mass exerted on the egg surface when the sensor head touches the egg, results from both the impact of the sensor head and the moving parts of the sensor head. Therefore, the mass of the moving parts of the sensor head needs to be addressed. The total density of the sensor head is then determined from the mass and volume. Calculation results show that the total density of the sensor head is approximately $4.79 \times 10^{4} \mathrm{~kg} / \mathrm{m}^{3}$, and that the density of the eggshell is give by $\rho_{2}$. Experiments on egg products indicate that the density of eggshells is approximately $1.18 \times 10^{3} \mathrm{~kg} / \mathrm{m}^{3}$; when the density of the two is filled into the equation above, and the test radius on the egg $\left(R_{2}\right)$ increased, then the product of $n$ and $n_{1}$ decreases relatively, creating a negative relationship. Equation A9 shows that the product of $n$ and $n_{1}$ is inversely proportional to the highest compress distance during collision $\left(\alpha_{1}\right)$. These findings indicate that increasing the test radius $\left(R_{2}\right)$ of the egg also increases the highest compression distance during collision, because of the relative rise in contact time. 


\section{References}

Ay, C., Guo, F.R., Lin, J.L. and Wan, Y.N. (1997). The study of eggshell's quality and crack detection by acoustics. Journal of National Chiayi Institute of Technology, 55, 27-41 (in Chinese).

Chang, J.C. (2003). Basic study on application of Hertz contact theory for detecting eggshell. Master Thesis, National Chung Hsing University, Taichung, Taiwan, R.O.C.

Chang, J.C., Chen, Y.C., Lee, H.T. and Cheng, C.W. (2003). Study on measuring the thickness of eggshells by using the accelerometer. Journal of Agriculture and Forestry, 52, 69-85 (in Chinese).

Chang, S.S. (1994). "Egg Science and Technology." pp. 67-75, $218-$ 224.

Cheng, C.W. (2004). Non-destructive quality detector for vegetables and eggshells. Taiwan Patent, Devise No. 205450.

Cho, H.K., Choi, W.K. and Paek, J.H. (2000). Detection of surface cracks in shell eggs by acoustic impulse method. Transactions of ASAE, 43, 1921-1926.

Coucke, P., Jacobs, G., Sas, P. and Baerdemaeker, J. De. (1998). Comparative analysis of the static and dynamic mechanical eggshell behaviour of a chicken egg. ISMA23 (International Conference on Noise and Vibration Engineering). 1497-1502.

C.O.A. (2008). Agriculture Statistics Yearbook. Council of Agriculture, Executive Yuan, R.O.C., 19.

Elster, R.T. and Goodrum, J.W. (1991). Detection of cracks in eggs using machine vision. Transactions of the ASAE, 34, 307-312.

Goodrum, J.W. and Elster, R.T. (1992). Machine vision for crack detection in rotating eggs. Transactions of the ASAE, 35, 13231328.

Ketelaere, B. De., Coucke, P. and Baerdemaeker, J. De. (2000). Eggshell crack detection based on acoustic resonance frequency analysis. Silsoe Research Institute, 76, 157-163.

Kitching, R. (1997). Pressured eggshell analysis. Int. J. Mech. Sci.,
39, 391-396.

Ko, S.C. (2001). An analysis of consumer preference for duck eggs in Taiwan. Master Thesis, Taichung, National Chung Hsing University.

Lin, D.K., Jee, W.M., Li, C.W. and Cheng, C.W. (2001). The study of relationship between the eggshells' crack locations and the detecting points. Journal of Agriculture and Forestry, 50, 57-78 (in Chinese).

Lin, J., Puri, V.M. and Anantheswaran, R.C. (1995). Measurement of eggshell thermal-mechanical properties. Transactions of the ASAE, 38, 1769-1776.

Lin, J., Puri, V.M. and Anantheswaran, R.C. (1996). Strains in eggshell during cooling of eggs-measurement and prediction using the finite element method. Transactions of the ASAE, 39, 10051012.

Narushin, V.G. (1997). Non-destructive measurements of egg parameters and quality characteristics. World's Poultry Science Journal, 53, 141-153.

Pai, S.Y. (2000). The study of eggshell's crack detection by acoustics. Master Thesis. National Chung Hsing University, Taichung, Taiwan, R.O.C.

Timoshenko, S.P. and Goodier, J.N. (1975). "Theory of Elasticity." McGraw-Hill Book Company, Third Edition, pp. 409-413.

Voisey, P.W., Hamilton, R.M.G. and Thompson, B.K. (1979). Laboratory measurements of eggshell strength. 2. The quasi-static compression, puncture, non-destructive deformation, and specific gravity methods applied to the same egg. Poultry Science, 58, 288-294.

Worley, J.W. and Goodrum, J.W. (1995). Strobe versus incandescent lighting for egg crack detection using machine vision. Transactions of the ASAE, 11, 549-554. 